\title{
Optical coherence tomography angiography in choroidal melanoma and nevus
}

This article was published in the following Dove Press journal:

Clinical Ophthalmology

\author{
Fariba Ghassemi ${ }^{1,2}$ \\ Reza Mirshahi ${ }^{1,2}$ \\ Kaveh Fadakar ${ }^{1,2}$ \\ Siamak Sabour ${ }^{3}$ \\ 'Retina \& Vitreous Service, ${ }^{2}$ Eye \\ Research Center, Farabi Eye Hospital, \\ Tehran University of Medical \\ Sciences, ${ }^{3}$ Safety Promotion and \\ Injury Prevention Research Centre, \\ Department of Clinical Epidemiology, \\ Shahid Beheshti University of Medical \\ Sciences, Tehran, Iran
}

Synopsis: In optical coherence tomography angiography, the choroidal vascular flow rate in choroidal melanoma is significantly lower than that in choroidal nevus.

Objective: The objective of this study was to describe the choriocapillaris and retinal features imaged by optical coherence tomography angiography (OCTA) in eyes with choroidal nevus from small malignant choroidal melanoma.

Methods: In this retrospective, noninvasive, observational study, 11 patients diagnosed with small choroidal mass (five with choroidal nevus and six with malignant melanoma) who underwent dilated fundus examination, ocular ultrasonography and OCTA images were compared.

Results: In choroidal nevus of all patients, OCTA demonstrated a hyporeflective mass with no significant deformity of choroidal vasculature and an intact retinal pigment epithelium (RPE)Bruch's membrane complex. The flow void mass was surrounded by an intense vascular rim named as surface microvasculature (SMV) that had an approximately similar flow rate median of $63.68 \mathrm{~mm}^{2}\left(60.42-67.62 \mathrm{~mm}^{2}\right)$, comparable with the median of the contralateral normal eye of $61.77 \mathrm{~mm}^{2}\left(60.42-64.53 \mathrm{~mm}^{2} ; P>0.09\right)$ for nevi. OCTA showed an obscured Bruch's membrane-RPE-Bruch's membrane complex and outer retinal layer in choroidal melanomas. Choriocapillaris flow rate over the melanomas was 55.73\% (41.93\%-60.82\%), and the corresponding normal areas had a flow area of $62.75 \%(61.99 \%-63.10 \% ; P=0.01)$. A flow rate difference between choroidal melanoma and nevus was significant $(P=0.006)$. Axial and peripheral feeding vessels were more dilated and tortuous compared with benign nevi.

Conclusion: Decreased flow rate of SMV of choroidal melanoma cases compared with nevi was a significant finding. Detection of characteristic vascular features of choroidal melanoma by OCTA could make OCTA an assuring diagnostic modality to differentiate malignant lesions.

Keywords: melanoma, choroidal nevus, OCT, OCTA, uveal melanoma

\section{Introduction}

Choroidal melanoma is a serious, life-threatening intraocular malignancy with risk for metastasis depending on tumor size. ${ }^{1}$ Small choroidal melanoma can be difficult to differentiate from choroidal nevus. Melanoma is believed to be a result of malignant transformation of choroidal nevus in some cases, and follow-up observations are needed for any alteration in the size. ${ }^{2}$ Although the risk for metastatic melanoma is likely to be related based on genetic profiles, it is also correlated with tumor size. Making the correct diagnosis of choroidal nevus protects the patients against the visually damaging effects of unnecessary treatment. In addition, when a small-sized melanoma is diagnosed in a timely manner, metastatic risk at 10 years is $12 \%$ compared with a medium-sized melanoma at 26\% and large-sized melanoma at 50\%. ${ }^{3}$ Many clinical and imaging findings have been described as predictive factors for differentiation between choroidal nevus and melanoma.
Correspondence: Fariba Ghassemi Eye Research Center, Farabi Eye Hospital, Tehran University of Medical Sciences, Qazvin Square, Tehran I3366I635I, Iran

Tel +98 2I 55421006

Fax +98 21 5541 6134

Email ghasemi_f@sina.tums.ac.ir 
The diagnosis and classification of a melanocytic choroidal tumor is made primarily by indirect ophthalmoscopy. Clinical-based risk factors can identify nevus at risk, allowing for early intervention for melanoma therapy. ${ }^{4,5}$ To obtain further diagnostic information, many imaging modalities are used, each with some limitations.

With the new optical coherence tomography (OCT) imaging, more in vivo histologic details of the tumors are identified. Enhanced depth imaging (EDI)-OCT of choroidal nevus shows smooth-surface topography, chronic overlying retinal pigment epithelium (RPE) and retinal degenerative findings, mostly photoreceptor atrophy. ${ }^{3-8}$ In comparison, EDI-OCT of choroidal melanoma generally shows gentle, domed-shaped, smooth-surface topography with relatively fresh subretinal fluid demonstrating bushy photoreceptors. Bushy photoreceptors could denote edematous photoreceptors or macrophages with lipofuscin on the posterior surface of the detached retina. ${ }^{9}$

With the development of new OCT techniques, quality dye-free angiography of choroidal and retinal vasculature is now available. This technique provides information about blood flow by comparing consecutive B-scans. The characteristics of choroidal melanoma and nevus are not completely described in optical coherence tomography angiography (OCTA). There is no single imaging modality that can currently reliably differentiate the nevus from small choroidal melanoma lesions.

The purpose of this study was to describe OCTA findings in five cases of choroidal nevus and six cases of malignant melanoma, using the B-scan OCTA via split-spectrum amplitude-decorrelation angiography (SSADA) and en face modality.

\section{Methods}

In this study, we retrospectively reviewed the records of patients with choroidal nevus and melanoma who were examined in the Department of Ophthalmology, Tehran University of Medical Sciences, Farabi Eye Hospital. An informed consent was obtained from all patients, and the ethics committee of Tehran University of Medical Sciences approved the study and its method. The exclusion criteria were a history of associated retinal microvascular abnormality such as diabetes, history of ocular trauma or glaucoma, previous laser photocoagulation or brachytherapy, refractive error $>3$ diopters, and patients in whom the lesions were far away from the arcades.

A thorough investigation including history taking and comprehensive examination were performed. Following slit-lamp examination, fundus photography and echography were obtained, and the size (using B-scan - Aviso, $10 \mathrm{MHz}$; Quantel Medical, Cournon d'Auvergne, France) and location of the tumor were recorded. Descriptive characteristics such as tumor thickness, subretinal fluid, retinal abnormalities at the level of RPE and retinal outer layers and lipofuscin pigment accumulation were documented. In this study, clinical features in combination with B-scan characteristics were used for diagnosis. Pathologic examination was not performed.

OCTA using the Avanti RTVue XR with AngioVue (version: 2016.1.0.23 - beta; OptoVue, CA, USA) was performed using $3 \times 3 \mathrm{~mm}, 6 \times 6 \mathrm{~mm}$ and $8 \times 8 \mathrm{~mm}$ sections for both eyes of each subject without pharmacologic pupillary dilation. We used the SSADA algorithm in this study. This instrument operates at $840 \mathrm{~nm}$ wavelength and performs 70,000 A-scans per second to acquire OCTA volumes consisting of two repeated B-scans of $3 \times 3 \mathrm{~mm}$ $(304 \times 304$ pixels $)$ in the transverse dimension. Two volumetric raster scans, including one horizontal priority (x-fast) and one vertical priority (y-fast), were obtained consecutively, and the merging of two scans removed many motion artifacts. Low-quality scans (ie, due to blinking or motion) were excluded and repeated until good quality scans were achieved. The foveal region was outlined as a central circle with a 120-pixel $(1.2 \mathrm{~mm})$ diameter, and the parafoveal region was delineated as a ring, by 91 pixels width, surrounding the foveal region. ${ }^{10}$ Foveal avascular zone (FAZ; superficial and deep) areas were measured in square millimeters. Briefly, a non-flow area measurement is preset on the Optovue instrument. Upon clicking on the center of the FAZ, the software automatically calculated the areas on the superficial and deep vascular network. ${ }^{11}$ The user manually fine-tuned the plane to maximize the visualization of the retinal capillary bed.

The OCTA images were semi-automatically segmented and evaluated for changes at the level of the choriocapillaris. The manual segmentation of the choriocapillaris was achieved by first viewing the choriocapillaris slab segmentation automatically generated by the AngioVue software. The position of this slab was manually corrected by scrolling up or down in the corresponding B-scan, and the slab thickness was adjusted to minimize decorrelation tail artifacts. To evaluate the vascularity of the choroidal mass, the OCTA image was segmented below the level of RPE through the choriocapillaris, and underneath bridging the inner surface of the tumor. Tumor area was manually depicted on the en face plane, and measurements were conducted. The OCTA commercial software calculated the flow area per $1 \mathrm{~mm}^{2}$ in $3 \times 3 \mathrm{~mm}$ scans in the choriocapillaris and choriocapillaris over the lesion. 
The flow rate percentage was calculated in $1 \mathrm{~mm}^{2}$ radius by dividing the measured flow area by the selected area and then multiplying by 100 on the $31-59 \mu \mathrm{m}$ section below the level of RPE over the lesion and the corresponding part of contralateral normal eye. Surface microvasculature (SMV) was defined as the bulk of a dense, compact vasculature covering the surface and around the tumor detected on OCTA cross-sectional images. Two trained readers (RM and FG) reviewed and graded all OCT images. The results were were agreed between two readers.

\section{Results}

Of 13 choroidal-pigmented lesions in 13 cases, only 11 lesions in 11 eyes were feasible for thorough study. Five had nongrowing choroidal nevus and six had growing choroidal melanomas detected after a mean of 21.3 months of follow-up (Figures 1 and 2 and Table 1). The small melanomas ranged from 1.5-3.6 mm, and all but one nevus was flat.

We noted that the elderly or uncooperative patients and the patients with media opacity, substantial amount of subretinal fluid, the mid-peripheral and peripheral location
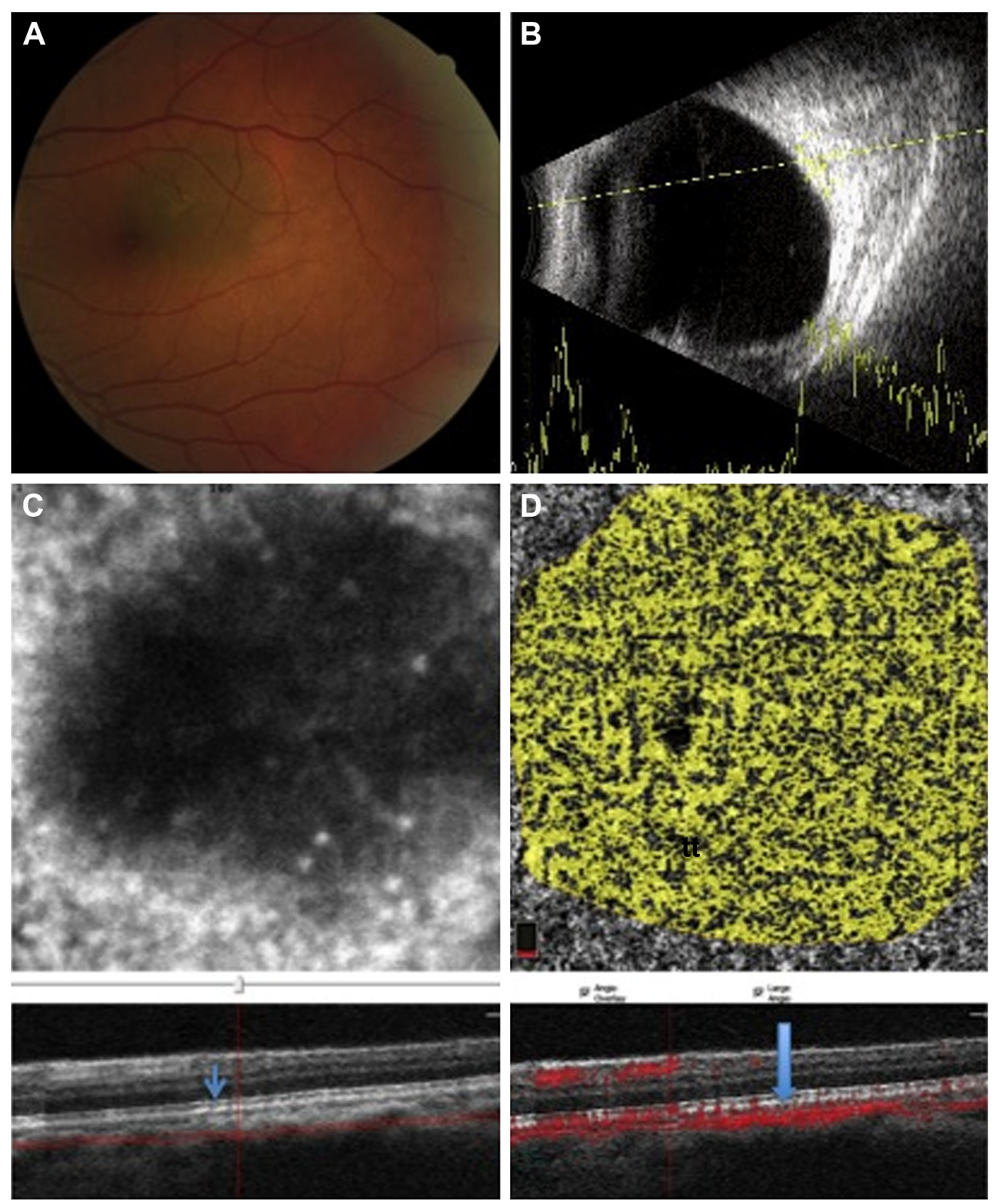

Figure I A case with a small choroidal nevus in the posterior pole of the left eye with some small drusen over the lesion.

Notes: (A) The fundus photograph of a pigmented lesion on the macular area without exudative changes. (B) B-scan showing a nearly flat, small choroidal lesion with medium-to-high internal reflectivity. (C) OCTA with slabs crossing the surface of the choroidal nevus at the choriocapillaris level by en face imaging with intact outer retina (arrow). (D) The flow rate over the lesion at the level of choriocapillaris level was evaluated (arrow). It elucidates more concentrated vasculature but is still not much different than nearby retina.

Abbreviation: OCTA, optical coherence tomography angiography. 

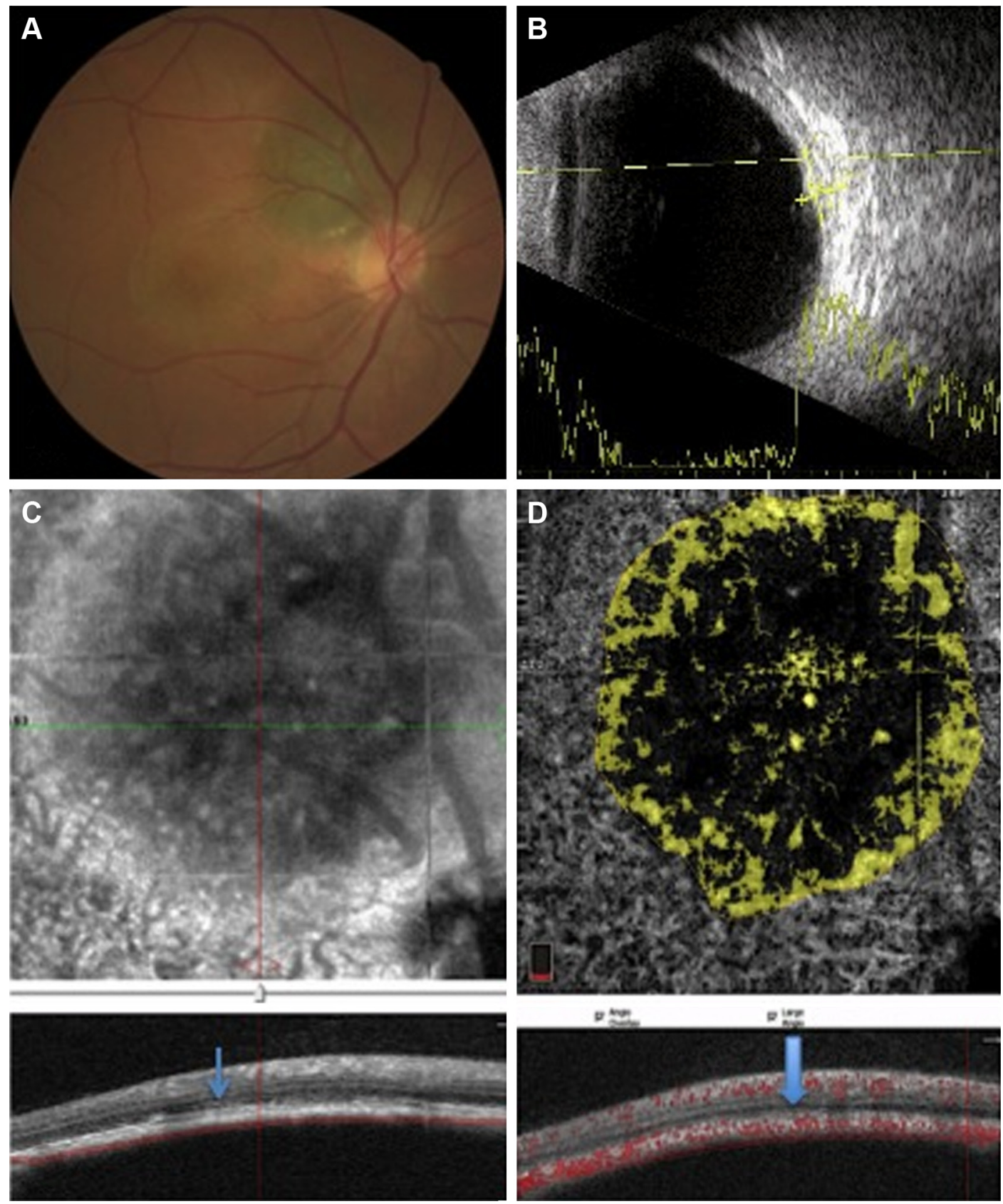

Figure 2 A case with a small and growing peripapillary choroidal melanoma.

Notes: (A) The fundus photograph demonstrates a pigmented superior peripapillary lesion and pigmentary changes in the macular area due to multiple episodes of increasing subretinal fluid. (B) B-scan shows a small choroidal lesion with medium-to-high internal reflectivity. (C) Cross-sectional B-scan OCTA with slabs crossing the surface of the choroidal melanoma through the choriocapillaris. The RPE-Bruch's membrane complex shows some derangement with subretinal fluid (arrow) on en face imaging. (D) The flow rate over the lesion at the level of choriocapillaris level was measured, showing thinner vascular area than the nearby choriocapillaris (arrow).

Abbreviations: OCTA, optical coherence tomography angiography; RPE, retinal pigment epithelium.

of the tumor and tumors with a thickness $>3.5 \mathrm{~mm}$ were not good candidates for OCTA. In three patients, the OCTA could not be used for the normal eye. All the tumors were a pigmented type of nevi and melanomas. Probably because of the high pigment content of the tumor, the vascular pattern details of the inside of both nevi and melanomas could not be appraised well (Table 2). OCTA images obviously showed more axial or peripheral feeding vessels in choroidal melanomas than nevi. However, for the melanomas, vascular flow rate over the lesion was lower than that over the normal contralateral counterpart at the choriocapillaris level (Table 3).

The mean thickness was $0.4 \mathrm{~mm}$ (median $0 \mathrm{~mm}$, range 0-2 $\mathrm{mm}$ ) in the nevi group and $2.35 \mathrm{~mm}$ (median $2.1 \mathrm{~mm}$, 
Table I Demographic findings of the patients with small melanocytic nevi and treatment-naive small choroidal melanomas

\begin{tabular}{|c|c|c|c|c|c|c|c|c|c|c|c|}
\hline \multirow[t]{2}{*}{ Characteristics } & \multicolumn{5}{|c|}{ Choroidal nevus } & \multicolumn{6}{|c|}{ Choroidal melanoma } \\
\hline & Case I & Case 2 & Case 3 & Case 4 & Case 5 & Case 6 & Case 7 & Case 8 & Case 9 & Case 10 & Case II \\
\hline Age (years) & 67 & 64 & 55 & 58 & 68 & 49 & 54 & 65 & 55 & 75 & 52 \\
\hline Sex & $M$ & $\mathrm{~F}$ & $\mathrm{~F}$ & $\mathrm{~F}$ & M & $M$ & $\mathrm{~F}$ & $M$ & M & $M$ & $\mathrm{~F}$ \\
\hline Eye & OD & OS & OD & OD & OD & OS & OD & OS & OD & OD & OD \\
\hline Symptom & - & + & + & + & + & + & + & + & + & + & + \\
\hline Visual acuity & $5 / 10$ & $2 / 10$ & $3 / 10$ & $10 / 10$ & $3 / 10$ & $5 / 10$ & $7 / 10$ & $3 / 10$ & $3 / 10$ & $5 / 10$ & $3 / 10$ \\
\hline Diameter (mm) & 2 & 5 & 2 & 7 & 5 & 5.3 & 6.54 & 8.7 & 8.5 & 5 & 8 \\
\hline Thickness (mm) & Flat & Flat & Flat & 2 & Flat & 1.8 & 1.5 & 3 & 3.6 & 2 & 2.23 \\
\hline Subretinal fluid & - & - & - & - & - & + & + & + & + & + & + \\
\hline Follow-up (months) & 24 & 20 & 20 & 21 & 22 & 22 & 24 & 19 & 24 & 20 & 19 \\
\hline
\end{tabular}

Abbreviations: $F$, female; $M$, male.

range $1.5-3.6 \mathrm{~mm}$ ) in the melanoma group. Nearly all nevi had intact outer retina and RPE-Bruch's membrane complex. On the other hand, a thick and deranged ellipsoid zone (EZ) and obscured interdigitation zone (IDZ) and RPE-Bruch's membrane complex in the retina over the choroidal melanomas were prominent findings on B-scan images (OCTA; Table 2). The inner retina contour was deranged in one case, with cystoid changes in another case and the retina was atrophic in the third case of the melanoma group. In the nevus group, inner retina was normal for all eyes. We were able to demonstrate the presence of mural and piercing feeding vessels that were hardly visible with other imaging techniques for the melanomas. In the nevi, the flow void mass was surrounded by a vascular rim.

Flow signal over both the nevi and melanomas was increased on B-scan; however; the flow rate over the melanoma lesions was far less than that over nevus lesion (Figures 1 and 2). The microvascular flow rate per $1 \mathrm{~mm}^{2}$ over the lesion at the choriocapillaris level on nevi was comparable to the flow rate of the corresponding location of the contralateral normal eye at the same level $(63.68 \%[60.42 \%$ $67.62 \%]$ vs $61.77 \%$ [60.42\%-64.53\%]; $P=0.09$ ). In choroidal melanomas, the microvascular flow rate over the lesion at the choriocapillaris level was $55.73 \%(41.93 \%-60.82 \%)$, which was lower than the flow rate of the corresponding contralateral normal eye area of $62.75 \%$ (61.99\%-63.10\%; $P=0.01)$. A flow rate difference in the choriocapillaris over the lesion in choroidal melanoma and nevus was statistically significant $(P=0.006$; Table 3$)$. The superficial and deep FAZs in the affected eyes of both groups were comparable with the corresponding FAZs of unaffected eyes (Table 3).

\section{Discussion}

With new OCTA imaging, we were able to demonstrate the presence of mural and piercing feeding vessels that were hardly visible with other imaging techniques. The flow rate over the melanoma lesions was far less than that over nevi.

There is no widely accepted gold standard of care for small melanomas or other indeterminate melanocytic lesions, and the management ranges from radiation to observation. The asymmetry, border, color and diameter $(\mathrm{ABCD})$ rule has a high predictive value for differentiation of dysplastic cutaneous nevi in their early phase. ${ }^{12}$ Similarly, TFSOM UHHD thickness $>2 \mathrm{~mm}$, fluid, symptoms, orange pigment, margin near disc, ultrasonographic hollowness, halo absence and drusen absence - remembered through the mnemonic "To find small ocular melanoma using helpful hints daily" are proposed as the most important clinical risk factors for differentiating early choroidal melanoma from nevus. ${ }^{13}$ However, overlapping features between choroidal nevus and small melanoma such as tumor thickness, related subretinal fluid and orange pigments still prohibit timely accurate diagnosis in some cases. Regarding this fact, additional characteristics such as vascular density and appearance of feeding vessels could be helpful, which underscores the role of OCTA in the early detection of choroidal melanoma. OCTA had some limitations in detecting the whole tumor vascular content details, probably due to high pigment content of these lesions visible with the laser wavelength used. Using the SSADA method of OCTA, the microvascular flow rate over choroidal melanoma was way less than that over nevus. The microvascular flow rate over the nevus was comparable to that over the normal counterpart of the contralateral eye. OCTA images obviously showed more axial or peripheral feeding vessels in choroidal melanomas than nevi. However for the melanomas, vascular flow rate over the lesion was lower than that over the normal contralateral counterpart at the choriocapillaris level.

There is a well established correlation between malignant transformation of nevus and its vascular density. The microvasculature density inside and around the tumor increases 


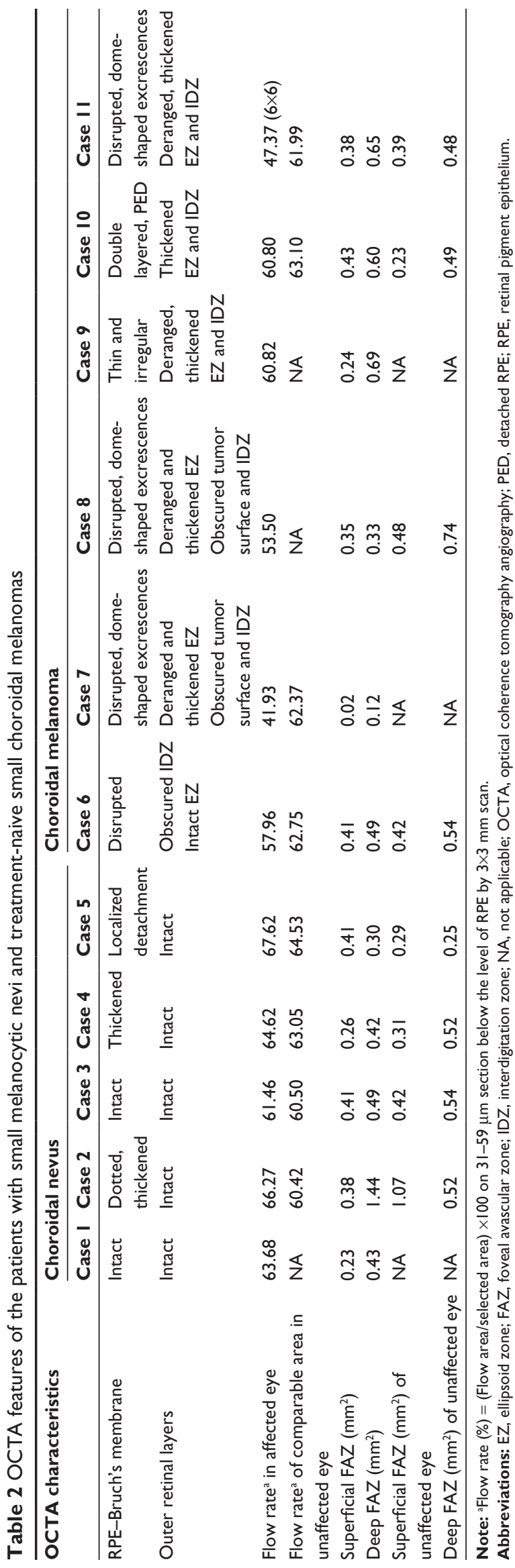

significantly in malignant melanoma compared to a benign nevus. ${ }^{14}$ Additionally, it is also an important prognostic factor that has direct association with tumor progression rate. ${ }^{15}$ De Carvalho et $\mathrm{al}^{16}$ disclosed in vivo microangiographic alterations in transformation of cutaneous nevus to melanoma by speckle-variance OCT (SV-OCT) in a single case. In the transversal section, SV-OCT showed vessels organized in larger vertical columns with irregular distribution. In the en face view, vascular pattern was characterized by many, densely packed dots progressively becoming irregular cloud-like structures with depth. Whereas, in nevus, the microvasculature consists of thin regular columns on the transversal section and regularly spread dots or short curved lines, progressively assuming a regular reticulated architecture with depth in the en face view. ${ }^{16}$

This finding highlights the vascular alteration as a crucial step in the early stage of transition of a nevus to melanoma. ${ }^{16}$

Dense, compact vasculature covering the surface of the lesion (SMV) was a common feature in both nevus and melanoma cases in our study. The probable source of this SMV on the top of the lesion was the compressed innate choriocapillaris network around the lesion, nourishing the lesion. Furthermore, superficial choroidal vessels on the top of melanomas seemed to be more reduced, causing less flow surface area over the lesion. This could be due to relentless compressive effect of the growing melanoma tumor in the choroid. However, the growth vector would be rationally more prominent toward the retina by lower resistance than underlying sclera.

Choroidal vessels were more prominent around and inside melanomas than nevi (Table 2). The signal void areas within the pigmented tumors in our cases are probably mainly because of shadowing effect and signal absorption by the melanin and/or lipofuscin, stacked in or on the tumor. Hence, the vascular network inside the tumor could not be elicited in detail. However, it is worth emphasizing that using the higher wavelength laser beam for imaging as in swept source imaging could be an advanced future step. Recently, Maloca et $a l^{17}$ have conducted a pilot study that evaluated the vascular network of small melanocytic choroidal tumors (maximum basal diameter of $6 \mathrm{~mm}$ and thickness of $1.5 \mathrm{~mm}$ ) with swept source OCT to illustrate speckle noise-free OCT images. Radial mural vessels entering the lesion were shown in a single reported nevus case and both cases of small melanoma, as radial or axial vessels. This finding prevented a comparison of benign mass with tumors with malignant features. ${ }^{17}$

In melanoma cases, on B-scan images (OCTA), the outer retina has some disorganization especially in the EZ and IDZ 
Table 3 OCTA FAZ and flow rate in the affected eye with melanoma or nevus versus the fellow eye in II consecutive cases

\begin{tabular}{|c|c|c|c|}
\hline OCTA characteristics & Nevus $(n=5)$ & Melanoma $(n=6)$ & $P$-value ${ }^{a}$ \\
\hline \multicolumn{4}{|l|}{ Affected eye } \\
\hline Superficial FAZ $\left(\mathrm{mm}^{2}\right)$, median ${ }^{\mathrm{b}}$ (range) & $0.38(0.23-0.4 I)$ & $0.37(0.02-0.43)$ & 0.926 \\
\hline Deep FAZ (mm²), median (range) & $0.43(0.30-1.44)$ & $0.55(0.12-0.69)$ & 0.784 \\
\hline Flow rate $\%(3 \mathrm{I}-59 \mu \mathrm{m})$, median (range) & $63.68(60.42-67.62)$ & $55.73(41.93-60.82)$ & 0.006 \\
\hline \multicolumn{4}{|l|}{ Non-affected eye } \\
\hline Superficial FAZ (mm²), median ${ }^{\mathrm{b}}$ (range) & $0.37(0.29-1.07)$ & $0.39(023-0.42)$ & 0.685 \\
\hline Deep FAZ $\left(\mathrm{mm}^{2}\right)$, median ${ }^{\mathrm{b}}$ (range) & $0.52(0.25-0.54)$ & $0.49(0.48-0.54)$ & 0.661 \\
\hline Flow rate $\%(3 \mathrm{I}-59 \mu \mathrm{m})$, median (range) & $61.77(60.42-64.53)$ & $62.75(61.99-63.10)$ & 0.773 \\
\hline$P$-value (affected vs nonaffected contralateral eye) & 0.09 & 0.01 & \\
\hline
\end{tabular}

Notes: aMann-Whitney $U$ test. 'Median is presented for small sample size.

Abbreviations: FAZ, foveal avascular zone; OCTA, optical coherence tomography angiography.

and RPE-Bruch's membrane complex. This could be caused by tumor compressive effects, retina and/or choroid related factors, or inflammatory mediators. Direct microinvasion of outer retina by tumor cells and other retinal ischemia due to long-standing subretinal fluid could be the cause. This is in accordance with a recent observation by Mashayekhi et $\mathrm{a}^{18}$ that eyes with treatment-naive melanoma commonly demonstrate subclinical mild macular edema.

In this study, the eyes with nevi and melanomas had comparable FAZ sizes with those of normal eyes. This is in contrast to recent studies showing larger FAZ size in melanoma-bearing eyes compared with the normal eyes..$^{19,20}$ They showed that this difference is a result of tumor-induced ischemia. ${ }^{18,19}$ This contradiction between these studies and ours could be due to different types of software used for measurements. Additionally, in those studies, large-sized tumors $(4.00 \mathrm{~mm})$ were evaluated and compared with mean $2.35 \mathrm{~mm}$ thick melanomas in our melanoma cases. In our nevus cases with the diameter of $0.4 \mathrm{~mm}$, no outer segment and FAZ involvement were observed.

This study is limited by the small number of cases and retrospective nature. We have no pathologic confirmation of melanoma versus nevus as a caveat in this study. We did not cover a wide range of melanomas but merely small-sized and posteriorly located tumors. Another limitation was that most of our analysis of OCT and OCTA characteristics of the tumors were qualitative, not quantitative. The nevi and melanomas with similar height were not compared head to head in terms of vascular pattern.

In conclusion our quantitative analysis showed lessened density of choroidal vasculature and flow over melanoma compared to nevus. The presence of mural and piercing vessels and subtle changes in the RPE-Bruch's membrane complex in melanoma were noted.

This could have resulted from the tendency of melanoma growth toward the RPE-Bruch's membrane complex and flattening of the choriocapillaris over the lesion and consequent derangement of outer retinal layers. Further larger studies are mandatory to provide reproducible results.

\section{Author contributions}

All authors made substantial contributions to conception and design, acquisition of data, or analysis and interpretation of data; took part in drafting the article or revising it critically for important intellectual content; gave final approval of the version to be published; and agree to be accountable for all aspects of the work.

\section{Disclosure}

The authors report no conflicts of interest in this work.

\section{References}

1. Shields CL, Manalac J, Das C, Ferguson K, Shields JA. Choroidal melanoma: clinical features, classification, and top 10 pseudomelanomas. Curr Opin Ophthalmol. 2014;25(3):177-185.

2. Singh AD, Kalyani P, Topham A. Estimating the risk of malignant transformation of a choroidal nevus. Ophthalmology. 2005;112(10): 1784-1789.

3. Shields CL, Furuta M, Thangappan A, et al. Metastasis of uveal melanoma millimeter-by-millimeter in 8033 consecutive eyes. Arch Ophthalmol. 2009;127(8):989-998.

4. Shields CL, Cater J, Shields JA, Singh AD, Santos MC, Carvalho C. Combination of clinical factors predictive of growth of small choroidal melanocytic tumors. Arch Ophthalmol. 2000;118(3):360-364.

5. Shields CL, Furuta M, Berman EL, et al. Choroidal nevus transformation into melanoma: analysis of 2514 consecutive cases. Arch Ophthalmol. 2009;127(8):981-987.

6. Shields CL, Mashayekhi A, Materin MA, et al. Optical coherence tomography of choroidal nevus in 120 patients. Retina. 2005;25(3): 243-252.

7. Sayanagi K, Pelayes DE, Kaiser PK, et al. 3D Spectral domain optical coherence tomography findings in choroidal tumors. Eur J Ophthalmol. 2010;21:271-275.

8. Singh AD, Belfort RN, Sayanagi K, Kaiser PK. Fourier domain optical coherence tomographic and auto-fluorescence findings in indeterminate choroidal melanocytic lesions. Br J Ophthalmol. 2010;94(4): 474-478

9. Shields CL, Kaliki S, Rojanaporn D, Ferenczy SR, Shields JA. Enhanced depth imaging optical coherence tomography of small choroidal melanoma: comparison with choroidal nevus. Arch Ophthalmol. 2012; 130(7):850-856. 
10. Shahlaee A, Samara WA, Hsu J, et al. In vivo assessment of macular vascular density in healthy human eyes using optical coherence tomography angiography. Am J Ophthalmol. 2016;165:39-46.

11. Savastano MC, Lumbroso B, Rispoli M. In vivo characterization of retinal vascularization morphology using optical coherence tomography angiography. Retina. 2015;35(11):2196-2203.

12. Nachbar F, Stolz W, Merkle T, et al. The ABCD rule of dermatoscopy: high prospective value in the diagnosis of doubtful melanocytic skin lesions. J Am Acad Dermatol. 1994;30(4):551-559.

13. Shields CL, Shields JA, Kiratli H, De Potter P, Cater JR. Risk factors for growth and metastasis of small choroidal melanocytic lesions. Ophthalmology. 1995;102(9):1351-1361.

14. Zidlik V, Brychtova S, Uvirova M, Ziak D, Dvorackova J. The changes of angiogenesis and immune cell infiltration in the intra- and peri-tumoral melanoma microenvironment. Int J Mol Sci. 2015;16(4):7876-7889.

15. Wen Y, Li J, Koo J, et al. Activation of the glutamate receptor GRM1 enhances angiogenic signaling to drive melanoma progression. Cancer Res. 2014;74(9):2499-2509.

16. De Carvalho N, Ciardo S, Cesinaro A, et al. In vivo micro-angiography by means of speckle-variance optical coherence tomography (SV-OCT) is able to detect microscopic vascular changes in naevus to melanoma transition. J Eur Acad Dermatol Venereol. 2015;30(10):e67-e68.
17. Maloca P, Gyger C, Hasler PW. A pilot study to image the vascular network of small melanocytic choroidal tumors with speckle noise-free 1050-nm swept source optical coherence tomography (OCT choroidal angiography). Graefes Arch Clin Exp Ophthalmol. 2016;254(6): 1201-1210.

18. Mashayekhi A, Schönbach E, Shields CL, Shields JA. Early subclinical macular edema in eyes with uveal melanoma: association with future cystoid macular edema. Ophthalmology. 2015;122(5):1023-1029.

19. Valverde-Megías A, Say EA, Ferenczy SR, Shields CL. Differential macular features on optical coherence tomography angiography in eyes with choroidal nevus and melanoma. Retina. 2017;37(4): 731-740.

20. Li Y, Say EA, Ferenczy S, Agni M, Shields CL. Altered parafoveal microvasculature in treatment-naive choroidal melanoma eyes detected by optical coherence tomography angiography. Retina. 2017;37(1): $32-40$.
Clinical Ophthalmology

\section{Publish your work in this journal}

Clinical Ophthalmology is an international, peer-reviewed journal covering all subspecialties within ophthalmology. Key topics include: Optometry; Visual science; Pharmacology and drug therapy in eye diseases; Basic Sciences; Primary and Secondary eye care; Patient Safety and Quality of Care Improvements. This journal is indexed on

\footnotetext{
Submit your manuscript here: http://www.dovepress.com/clinical-ophthalmology-journal
}

\section{Dovepress}

PubMed Central and CAS, and is the official journal of The Society of Clinical Ophthalmology (SCO). The manuscript management system is completely online and includes a very quick and fair peer-review system, which is all easy to use. Visit http://www.dovepress.com/ testimonials.php to read real quotes from published authors. 